\title{
Tracking of objective physical activity and physical fitness in Japanese children
}

\author{
Kensaku Sasayama ${ }^{1 *}$ (1) and Minoru Adachi ${ }^{2}$
}

\begin{abstract}
Objective: The purpose of this study was to examine the tracking of objective physical activity and physical fitness from childhood to adolescence in Japanese children. The longitudinal study comprised 368 participants (aged 9-10 years) in 2008, and the study involved 134 participants (aged 13-14 years, a dropout rate of 63.6\%) in 2011. After excluding participants with missing data, a total of 111 participants (46 boys and 65 girls) were available for study. Step counts and moderate-to-vigorous physical activity (MVPA) were measured using a uniaxial accelerometer. Physical fitness was assessed using the following tests: hand grip, sit-ups, sit and reach, side-to-side steps, 20-m shuttle run, 50-m dash, standing broad jump and ball throwing.

Results: In boys, there was a significant correlation between objective physical activity and all physical fitness tests at baseline and follow-up. In girls, although there was no significant correlation between objective physical activity at baseline and follow-up, all physical fitness tests at baseline and follow-up were significantly correlated. In conclusion, moderate tracking was shown in objective physical activity of boys from childhood to adolescence. In addition, moderate to high tracking was shown in physical fitness of both sexes from childhood to adolescence.
\end{abstract}

Keywords: Longitudinal, Youth, Moderate-to-vigorous physical activity, Fitness, Asian children

\section{Introduction}

Several studies have reported that physical activity in childhood is correlated to both physical and mental aspects $[1,2]$. Therefore, the World Health Organization recommends that children aged 5-17 years age should accumulate at least $60 \mathrm{~min}$ of moderate-to-vigorous physical activity (MVPA) every day [3]. Similarly, the physical activity guidelines in the UK [4], USA [5] and Canada [6] also recommend this level of activity for similar age groups.

A review by Telema et al. [7] reported that physical activity tracks from childhood to adolescence and indicated that most previous studies used questionnaires to measure and assess physical activity. A questionnaire can easily evaluate physical activity and has the merit of low cost. However, previous studies [8] also reported that questionnaires overestimate physical activity

\footnotetext{
*Correspondence: sasayama@ped.ous.ac.jp

1 Faculty of Education, Okayama University of Science, 1-1, Ridai-cho, Kita-ku, Okayama 700-0005, Japan

Full list of author information is available at the end of the article
}

compared to accelerometry. In addition, Chinapaw et al. [9] reported that the reliability of physical activity questionnaires for children (mean age 6-12 years) is lower than that for adolescents (mean age 12-18 years). Therefore, it appears to be important to examine the tracking of physical activity using objective methods such as an accelerometer.

Physical fitness as well as physical activity is reportedly related to physical and mental aspects [10]. Malina [11] examined the tracking of physical fitness (e.g. strength, flexibility and endurance) and reported that physical fitness tracks from childhood to adolescence at low to moderate levels. To the best of our knowledge, the tracking of objective physical activity and physical fitness from childhood to adolescence has neither been measured nor reported in Japanese children. Therefore, it is necessary to confirm the tracking of physical activity and physical fitness from childhood to adolescence to promote the importance of physical activity and physical fitness in this population.

The purpose of this study was to examine the tracking of objective physical activity and physical fitness 
from childhood to adolescence in Japanese children. We hypothesised that objective physical activity and physical fitness track from childhood to adolescence in Japanese children.

\section{Main text \\ Methods \\ Study design and participants}

This study was conducted in Ibara City within the Okayama Prefecture of Japan during 2008-2011 and encompassed all 13 public elementary schools within the city. The first study comprised 368 participants aged 9-10 years (90.6\% within the city) in September 2008, and the follow-up study involved 134 participants (aged 13-14 years, with dropout rate of 63.6\%) in September 2011. After excluding participants with missing data, a total of 111 participants ( 46 boys, 65 girls) were included in the study.

\section{Physical activity}

Total steps and MVPA were measured using a uniaxial accelerometer (Kenz Lifecorder EX (LC); Suzuken Co. Ltd, Nagoya, Japan). Kumahara et al. [12] have previously reported that this accelerometer samples acceleration at a rate of 32 samples/second and assesses values ranging from 0.06 to $1.94 \mathrm{~g}$. The acceleration signal was filtered using an analogue bandpass filter and subsequently digitised. The maximum pulse over $4 \mathrm{~s}$ was measured as the acceleration value and classified into 11 activity levels $(0$, 0.5 and 1-9). The epoch length of the LC device is $2 \mathrm{~min}$. Sasayama and Adachi [13] showed that the activity level detected using an LC device was significantly correlated with metabolic equivalents (METs) during walking and running in Japanese children $(\mathrm{r}=0.883, \mathrm{p}<0.05)$. They confirmed that the activity level for MVPA ( $\geq 3$ METs) is equivalent to a value of $\geq 5$ as detected by the LC device; accordingly, the MVPA cut-off point was based on this finding [13]. Participants wore the LC device on their waists for 5 consecutive weekdays, at all times, excluding while sleeping, swimming, bathing or contact sports. Based on previous studies [14], accelerometer data were collected on at least 3 weekdays. A valid day was defined as at least $600 \mathrm{~min}$ of wear time during weekdays [15]. Non-wearing time was defined by at least $60 \mathrm{~min}$ of zero consecutive counts [16].

\section{Physical fitness}

Physical fitness was assessed using the new National Statistical Survey on Physical Fitness and Motor Ability by the Ministry of Education, Culture, Sports, Science and Technology (MEXT) in Japan (MEXT, 2000). The physical fitness test by MEXT was conducted as follows and test items included hand grip (muscle strength), sit-ups (abdominal strength and endurance), sit and reach (flexibility), side-to-side jump (agility), 20-m shuttle run (cardiorespiratory endurance), 50-m dash (speed), standing broad jump (explosive leg strength) and softball (9-10 years)/handball (13-14 years) throwing (explosive arm strength and throwing ability).

\section{Anthropometry}

Height (precision within $0.1 \mathrm{~cm}$ ) and body weight (precision within $0.1 \mathrm{~kg}$ ) were measured in light clothing without shoes. Body mass index (BMI) was calculated using the ratio of weight $(\mathrm{kg})$ to height squared $\left(\mathrm{m}^{2}\right)$.

\section{Statistical analysis}

Participant characteristics, physical activity and physical fitness variables were reported as mean \pm standard deviations. Characteristic differences in baseline and follow-up were analysed using paired Student's $t$ test. The associations between the obtained variables were analysed using Spearman correlation coefficient. Values ranging from 0.00 to 0.29 indicate low correlation, from 0.30 to 0.59 indicate moderate correlation and from 0.60 to 1.00 indicate high correlation [11]. All analyses were performed using SPSS Statistics software version 24. Results were considered statistically significant at $\mathrm{p}<0.05$.

\section{Results}

\section{Characteristics of participants at baseline and follow-up}

Characteristics of participants at baseline and followup are shown in Table 1. For both sexes, all variables of anthropometry, physical activity and physical fitness showed significant differences between baseline and follow-up.

\section{Tracking of physical activity and physical fitness}

Tracking of physical activity and physical fitness expressed as Spearman correlation coefficients are shown in Table 2. In boys, there was a significant correlation between total steps, MVPA, hand grip, sit-ups, sit and reach, side-to-side jump, 20-m shuttle run, 50-m dash, standing broad jump and ball throwing at baseline and follow-up. In girls, although there was no significant correlation between total steps and MVPA at baseline and follow-up, hand grip, sit-ups, sit and reach, side-to-side jump, 20-m shuttle run, 50-m dash, standing broad jump and ball throwing at baseline and follow-up were significantly correlated.

\section{Discussion}

The objective evaluation of physical activity by an accelerometer was a notable strength of the study. Adachi et al. [17] have previously reported that the total energy expenditure assessed using the doubly-labelled water 
Table 1 Participants of characteristics

\begin{tabular}{|c|c|c|c|c|c|c|}
\hline & \multicolumn{3}{|l|}{ Boys $(n=46)$} & \multicolumn{3}{|l|}{ Girls $(n=65)$} \\
\hline & Baseline (2008) & Follow-up (2011) & p value & Baseline (2008) & Follow-up (2011) & $\mathrm{p}$ value \\
\hline Age (years) & $9.2 \pm 0.4$ & $13.2 \pm 0.4$ & $<0.001$ & $9.1 \pm 0.3$ & $13.1 \pm 0.3$ & $<0.001$ \\
\hline Height (cm) & $134.1 \pm 5.8$ & $153.1 \pm 8.2$ & $<0.001$ & $135.1 \pm 5.4$ & $152.0 \pm 4.1$ & $<0.001$ \\
\hline Weight (kg) & $30.7 \pm 7.0$ & $44.4 \pm 10.2$ & $<0.001$ & $29.8 \pm 4.5$ & $43.9 \pm 6.3$ & $<0.001$ \\
\hline BMI $\left(\mathrm{kg} / \mathrm{m}^{2}\right)$ & $16.9 \pm 2.9$ & $18.8 \pm 3.0$ & $<0.001$ & $16.2 \pm 1.7$ & $19.0 \pm 2.4$ & $<0.001$ \\
\hline Total steps (steps/day) & $18387.7 \pm 3876.4$ & $12188.5 \pm 4872.9$ & $<0.001$ & $14627.3 \pm 3340.2$ & $11863.4 \pm 3153.3$ & $<0.001$ \\
\hline MVPA (min/day) & $60.6 \pm 18.5$ & $37.3 \pm 22.0$ & $<0.001$ & $39.9 \pm 13.5$ & $32.8 \pm 14.3$ & 0.004 \\
\hline Hand grip (kg) & $14.9 \pm 3.0$ & $23.4 \pm 6.9$ & $<0.001$ & $14.0 \pm 3.3$ & $21.8 \pm 4.6$ & $<0.001$ \\
\hline Sit-ups (count) & $19.3 \pm 5.7$ & $24.2 \pm 6.2$ & $<0.001$ & $14.6 \pm 3.9$ & $18.7 \pm 3.9$ & $<0.001$ \\
\hline Sit and reach $(\mathrm{cm})$ & $36.5 \pm 8.7$ & $40.2 \pm 8.5$ & 0.015 & $37.2 \pm 7.7$ & $42.4 \pm 7.1$ & $<0.001$ \\
\hline Side-to-side jump (count) & $40.5 \pm 7.0$ & $48.9 \pm 5.8$ & $<0.001$ & $37.7 \pm 5.6$ & $43.4 \pm 3.9$ & $<0.001$ \\
\hline 20-m shuttle run (count) & $45.7 \pm 16.8$ & $68.1 \pm 20.9$ & $<0.001$ & $29.9 \pm 11.6$ & $46.0 \pm 12.8$ & $<0.001$ \\
\hline 50-m dash (s) & $9.4 \pm 0.7$ & $9.0 \pm 0.9$ & $<0.001$ & $9.7 \pm 0.7$ & $9.3 \pm 0.7$ & $<0.001$ \\
\hline Standing broad jump (cm) & $147.6 \pm 14.7$ & $174.7 \pm 20.7$ & $<0.001$ & $141.1 \pm 16.1$ & $156.9 \pm 25.4$ & $<0.001$ \\
\hline Ball throwing $(\mathrm{m})$ & $24.6 \pm 6.8$ & $19.6 \pm 4.5$ & $<0.001$ & $13.7 \pm 5.1$ & $11.4 \pm 3.4$ & $<0.001$ \\
\hline
\end{tabular}

Values are means \pm standard deviations

BMI: Body mass index; MVPA: moderate-to-vigorous physical activity

$\mathrm{p}<0.05$ for different baseline and follow-up

Table 2 Tracking of physical activity and physical fitness expressed as Spearman correlation coefficients

\begin{tabular}{|c|c|c|c|c|}
\hline & \multicolumn{2}{|c|}{ Boys $(n=46)$} & \multicolumn{2}{|c|}{ Girls $(n=65)$} \\
\hline & $\mathbf{r}$ & p value & $\mathbf{r}$ & $p$ value \\
\hline \multicolumn{5}{|l|}{ Physical activity } \\
\hline Total steps (steps/day) & 0.319 & 0.031 & 0.178 & 0.156 \\
\hline MVPA (min/day) & 0.352 & 0.017 & 0.198 & 0.114 \\
\hline \multicolumn{5}{|l|}{ Physical fitness } \\
\hline Hand grip (kg) & 0.520 & $<0.001$ & 0.680 & $<0.001$ \\
\hline Sit-ups (count) & 0.588 & $<0.001$ & 0.575 & $<0.001$ \\
\hline Sit and reach $(\mathrm{cm})$ & 0.341 & 0.020 & 0.303 & 0.014 \\
\hline Side-to-side jump (count) & 0.470 & $<0.001$ & 0.444 & $<0.001$ \\
\hline 20-m shuttle run (count) & 0.618 & $<0.001$ & 0.513 & $<0.001$ \\
\hline 50-m dash (s) & 0.736 & $<0.001$ & 0.734 & $<0.001$ \\
\hline Standing broad jump (cm) & 0.548 & $<0.001$ & 0.602 & $<0.001$ \\
\hline Ball throwing (m) & 0.647 & $<0.001$ & 0.549 & $<0.001$ \\
\hline
\end{tabular}

MVPA: Moderate-to-vigorous physical activity

method was significantly correlated with total steps and activity level detected using an accelerometer in Japanese children. In Japanese children aged 9-13 years, we found moderate tracking of physical activity (only boys) and moderate to high tracking of physical fitness (both boys and girls).

Previous studies [18-21] measuring total physical activity using pedometers or accelerometers have reported that total physical activity tracks from childhood to adolescence at low to moderate levels. Although these studies differ from the current study in terms of the ages of participants, follow-up period and pedometer or accelerometer use, the present study also showed moderate tracking for total steps in boys (Table 2). On the other hand, in our study, tracking was not confirmed for total steps in girls (Table 2). In some previous studies, tracking of total physical activity in both boys and girls reported similar results [20] but some studies $[19,21]$ showed that high tracking of total physical activity was confirmed in boys compared to girls. Therefore, there are possibilities of gender differences in terms of tracking. This gender difference may be influenced by the fact that total physical activity in girls is lower than that of boys from childhood to adolescence. Indeed, Wolff-Hughes et al. [22] reported that total physical activity in boys was higher than that in girls, regardless of age (6-19 years). WolffHughes et al. [22] also reported that total physical activity in girls tends to sharply decrease at adolescence compared to boys. This phenomenon may influenced tracking from childhood to adolescence.

Previous studies $[18,23-26]$ have reported that MVPA using accelerometer tracks from childhood to adolescence at low to moderate levels. We confirmed that MVPA tracks moderately only in boys from childhood to adolescence (Table 2). It has been reported that MVPA tracking is more robust in girls than in boys [24]. On the other hand, another report showed that MVPA tracking was similar for boys and girls [25]. Therefore, for MVPA as well as total steps, it is necessary to confirm whether there is gender difference in tracking. 
Regarding the tracking of physical fitness, it has been reported elsewhere [11] that physical fitness, such as muscular strength and endurance, power or explosive strength, running speed, agility, cardiovascular fitness track at low to moderate levels from childhood to adolescence. In our study, low to high tracking was confirmed in various physical fitness components (Table 2). Therefore, it was suggested that various physical fitness components track from childhood to adolescence in Japanese children.

\section{Conclusion}

Our results suggest that objective physical activity in Japanese boys track moderately from childhood to adolescence. In addition, for both Japanese boys and girls, physical fitness track moderately to highly from childhood to adolescence.

\section{Limitations}

Our study has several limitations. First, this study comprised a small sample size, had a short tracking term and was conducted only in the Okayama Prefecture in Japan. Since the study was conducted only in the Okayama Prefecture in Japan, its results cannot be applied to other populations or regions. Second, there were no controls for maturity related to physical fitness. Further studies are required with larger sample sizes and including participants from other populations. In addition, it is necessary to examine the maturity level.

\section{Abbreviations \\ MVPA: moderate-to-vigorous physical activity; LC: lifecorder; METs: metabolic equivalents; MEXT: Ministry of Education, Culture, Sports, Science and Technol- ogy; BMl: body mass index.}

\section{Acknowledgements}

We would like to thank the children and parents who participated in this study as well as the teachers for their support.

\section{Authors' contributions}

MA designed the study. MA and KS collected and analysed data. KS wrote the manuscript. MA critically reviewed the manuscript. Both authors read and approved the final manuscript.

\section{Funding}

No funding was provided.

\section{Availability of data and materials}

Please contact author for data requests.

\section{Ethics approval and consent to participate}

All participating children and their parents provided written informed consent before participation. The study was approved by the Institutional Review Board of Okayama University.

\section{Consent for publication}

Not applicable.

\section{Competing interests}

The authors declare that they have no competing interests.

\section{Author details}

${ }^{1}$ Faculty of Education, Okayama University of Science, 1-1, Ridai-cho, Kita-ku, Okayama 700-0005, Japan. ${ }^{2}$ Graduate School of Education, Okayama University, 3-1-1, Tsushima-naka, Kita-ku, Okayama 700-8530, Japan.

Received: 12 February 2019 Accepted: 30 April 2019

Published online: 07 May 2019

\section{References}

1. Janssen I, Leblanc AG. Systematic review of the health benefits of physical activity and fitness in school-aged children and youth. Int J Behav Nutr Phys Act. 2010;7:40.

2. Strong WB, Malina RM, Blimkie CJ, Daniels SR, Dishman RK, Gutin B, et al. Evidence based physical activity for school-age youth. J Pediatr. 2005;146(6):732-7.

3. World Health Organization. Global recommendations on physical activity for health. Geneva: WHO Press; 2010.

4. Department of Health and Social Care. Start active, stay active: report on physical activity in the UK 2011. https://www.gov.uk/government/publi cations/start-active-stay-active-a-report-on-physical-activity-from-thefour-home-countries-chief-medical-officers. Accessed 1 Mar 2019.

5. U.S. Department of Health and Human Services. Physical activity guidelines for Americans: be active, healthy, and happy! 2008. http://www.healt hgov/paguidelines/guidelines/defaultaspx. Accessed 1 Mar 2019.

6. Tremblay MS, Carson V, Chaput JP, Connor Gorber S, Dinh T, Duggan M, et al. Canadian 24-hour movement guidelines for children and youth: an integration of physical activity, sedentary behaviour, and sleep. Appl Physiol Nutr Metab. 2016;41(6 Suppl 3):S311-27.

7. Telama R. Tracking of physical activity from childhood to adulthood: a review. Obes Facts. 2009;2(3):187-95.

8. Lee PH, Macfarlane DJ, Lam TH, Stewart SM. Validity of the International Physical Activity Questionnaire Short Form (IPAQ-SF): a systematic review. Int J Behav Nutr Phys Act. 2011:8:115.

9. Chinapaw MJ, Mokkink LB, van Poppel MN, van Mechelen W, Terwee CB. Physical activity questionnaires for youth: a systematic review of measurement properties. Sports Med. 2010;40(7):539-63.

10. Ortega FB, Ruiz JR, Castillo MJ, Sjostrom M. Physical fitness in childhood and adolescence: a powerful marker of health. Int J Obes. 2008;32(1):1-11.

11. Malina RM. Tracking of physical activity and physical fitness across the lifespan. Res Q Exerc Sport. 1996;67(3 Suppl):S48-57.

12. Kumahara H, Schutz Y, Ayabe M, Yoshioka M, Yoshitake Y, Shindo M, et al. The use of uniaxial accelerometry for the assessment of physical-activityrelated energy expenditure: a validation study against whole-body indirect calorimetry. Br J Nutr. 2004;91 (2):235-43.

13. Sasayama K, Adachi M. Association between activity level assessed by a uniaxial accelerometer and metabolic equivalents during walking and running in male youths. J Phys Fit Sports Med. 2016;65(2):265-72.

14. Mattocks C, Ness A, Leary S, Tilling K, Blair SN, Shield J, et al. Use of accelerometers in a large field-based study of children: protocols, design issues, and effects on precision. J Phys Act Health. 2008;5(Suppl 1):S98-111.

15. Colley R, Connor Gorber S, Tremblay MS. Quality control and data reduction procedures for accelerometry-derived measures of physical activity. Health Rep. 2010;21(1):63-9.

16. Aadland E, Andersen LB, Anderssen SA, Resaland GK. A comparison of 10 accelerometer non-wear time criteria and logbooks in children. BMC Public Health. 2018;18(1):323.

17. Adachi M, Sasayama K, Hikihara Y, Okishima K, Mizuuchi H, Sunami Y, et al. Assessing daily physical activity in elementary school students used by accelerometer: avalidation study against doubly labeled water method. J Phys Fit Sports Med. 2007;56(3):347-55.

18. Basterfield L, Adamson AJ, Frary JK, Parkinson KN, Pearce MS, Reilly JJ. Longitudinal study of physical activity and sedentary behavior in children. Pediatrics. 2011;127(1):e24-30.

19. Nyberg G, Ekelund U, Marcus C. Physical activity in children measured by accelerometry: stability over time. Scand J Med Sci Sports. 2009;19(1):30-5.

20. Kristensen PL, Moller NC, Korsholm L, Wedderkopp N, Andersen LB, Froberg K. Tracking of objectively measured physical activity from childhood 
to adolescence: the European youth heart study. Scand J Med Sci Sports. 2008;18(2):171-8.

21. Raustorp A, Svenson K, Perlinger T. Tracking of pedometer-determined physical activity: a 5-year follow-up study of adolescents in Sweden. Pediatr Exerc Sci. 2007;19(2):228-38.

22. Wolff-Hughes DL, Bassett DR, Fitzhugh EC. Population-referenced percentiles for waist-worn accelerometer-derived total activity counts in U.S. youth: 2003-2006 NHANES. PloS ONE. 2014;9(12):e115915.

23. Raask T, Konstabel K, Maestu J, Latt E, Jurimae T, Jurimae J. Tracking of physical activity in pubertal boys with different BMI over two-year period. J Sports Sci. 2015;33(16):1649-57.

24. Francis SL, Morrissey JL, Letuchy EM, Levy SM, Janz KF. Ten-year objective physical activity tracking: lowa bone development study. Med Sci Sports Exerc. 2013;45(8):1508-14.
25. Kwon S, Janz KF. Tracking of accelerometry-measured physical activity during childhood: ICAD pooled analysis. Int J Behav Nutr Phys Act. 2012;9:68.

26. Baggett CD, Stevens J, McMurray RG, Evenson KR, Murray DM, Catellier DJ, et al. Tracking of physical activity and inactivity in middle school girls. Med Sci Sports Exerc. 2008;40(11):1916-22.

\section{Publisher's Note}

Springer Nature remains neutral with regard to jurisdictional claims in published maps and institutional affiliations.
Ready to submit your research? Choose BMC and benefit from:

- fast, convenient online submission

- thorough peer review by experienced researchers in your field

- rapid publication on acceptance

- support for research data, including large and complex data types

- gold Open Access which fosters wider collaboration and increased citations

- maximum visibility for your research: over $100 \mathrm{M}$ website views per year

At BMC, research is always in progress.

Learn more biomedcentral.com/submissions 